\title{
In Situ TEM Observation of Domain Nucleation and Domain Wall-Defect Interactions in Bismuth Ferrite Under Applied DC Bias
}

\author{
C.R. Winkler, ${ }^{*}$ L.W. Martin, ${ }^{* *}$ C.L. Johnson, ${ }^{*}$ and M.L. Taheri* \\ * Department of Materials Science \& Engineering, Drexel University, Philadelphia, PA 19104 \\ ** Department of Materials Science and Engineering, University of Illinois at \\ Urbana-Champaign, Urbana, IL 61801
}

Select multiferroic materials exhibit ferroelectric and magnetic order, and the two order parameters are coupled through a quantum-mechanical exchange interaction [1,2]. One of the most widely studied magneto-electric multiferroics is the perovskite $\mathrm{BiFeO}_{3}(\mathrm{BFO})$. The magneto-electric coupling in $\mathrm{BFO}$ allows control of the ferroelectric and magnetic domain structures via applied electric fields. Furthermore, BFO films possess high Néel and Curie temperatures and large ferroelectric polarization. Because of these unique properties, BFO and other magneto-electric multiferroics constitute a promising class of materials for incorporation into devices such as high-density ferroelectric and magnetoresistive memories, spin valves, and magnetic field sensors [3,4]. Before BFO can be integrated into devices, however, a full understanding of its ferroelectric and antiferromagnetic domain behavior across a range of time and length scales needs to be developed [1]. In situ TEM is one experimental technique capable of investigating the dynamics of ferroelectric domain behavior across a wide range of length scales.

We employ a custom-built biasing holder which permits the application of a DC bias through a set of epitaxial in-plane electrodes in the BFO thin film. By altering the magnitude and the polarity of the DC bias, we can control the magnitude and switch the direction of the in-plane electric field. Under an applied field, domains with ferroelectric polarization aligned with the field will increase in size, and this growth is accompanied by necessary domain wall propagation. In areas of large strain fields, such as those near dislocations, nucleation of a domain with polarization aligned with the applied field can occur. We investigate in situ the dynamic phenomena of ferroelectric domain nucleation and propagation. We capture these dynamic events using digital streaming video at $30 \mathrm{~Hz}$ across a range of relevant length scales and extract domain kinetics and nucleation energies from the resulting videos. A combination of in situ biasing and high-resolution transmission electron microscopy (HRTEM) allows us to study the motion of domain walls at the atomic level.

We will present multi-scale in situ biasing TEM experiments. Biasing experiments were carried out at medium magnifications (Figure 1) to study domain nucleation and propagation, from which we extracted quantitative thermodynamic data. Experiments were also performed at high magnifications where we studied how domain walls move through line defects (Figure 2), and how domain walls interact during collisions with other domain walls (Figure 3). These in situ HRTEM studies provide dynamic, direct experimental evidence of the behavior of domain-defect interactions at the atomic level. The results of our experiments will be correlated with theoretical and computational models to better understand the mechanisms of domain-defect interactions and their effects on the ferroelectric polarization in $\mathrm{BFO}$ and other ferroelectrics $[5,6,7]$. 
References

[1] R. Ramesh \& N.A. Spaldin, Nature Mater. 6 (1), (2007) 21.

[2] C. Ederer \& N.A. Spaldin, Curr. Opin. Solid State Mater. Sci. 9 (3), (2005) 128.

[3] Y.H. Chu et al., Nature Mater. 7 (8), (2008) 678.

[4] N.A. Spaldin \& M. Fiebig, Science 309 (5733), (2005) 391.

[5] A. Kontsos \& C.M. Landis, Int. J. Solids Struct. 46 (2009) 1491.

[6] Y. Shin, et al., Nature 449, (2007) 881.

[7] This research was made possible by use of the Centralized Research Facilities in the College of Engineering at Drexel University. This work was supported by the Department of Education and Drexel University through the GAANN-DREAM fellowship under contract P200A060117.

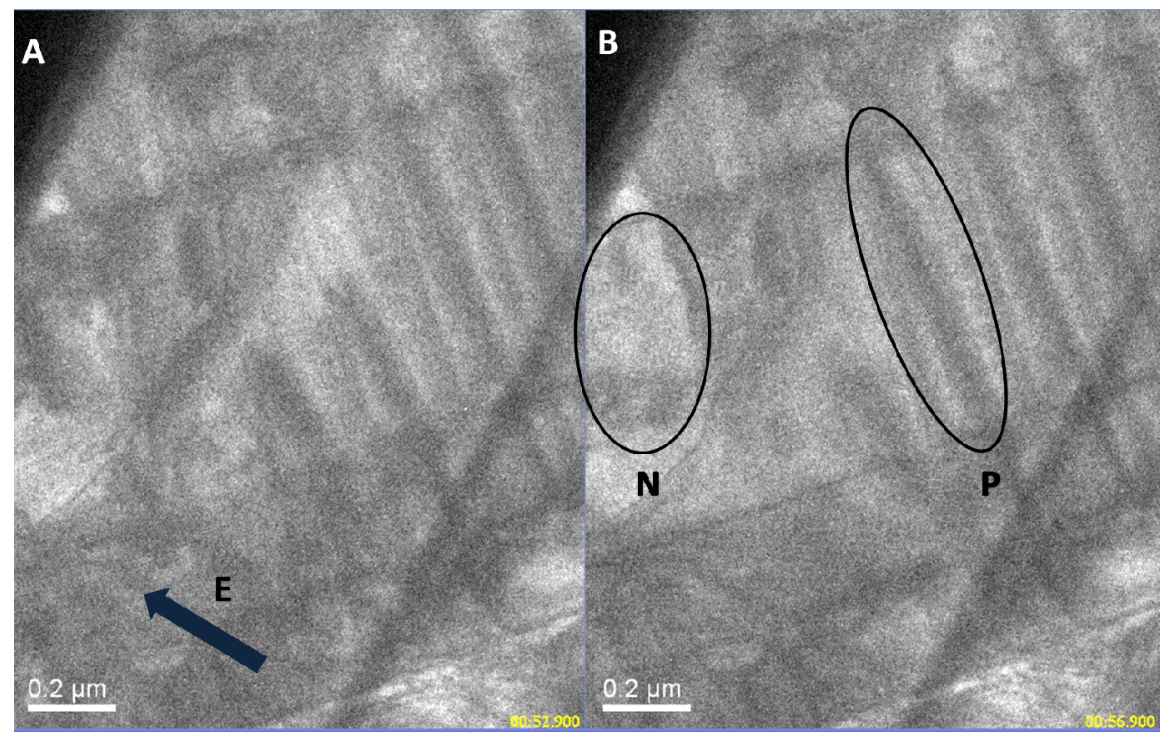

Figure 1: Bright field TEM micrograph showing a change in the ferroelectric domain structure of a BFO thin film under DC bias. In A) the direction of the in-plane electric field is indicated. In B) the ovals indicate areas of marked evolution of the ferroelectric domain structure, where " $N$ " marks a nucleation event and " $P$ " marks the propagation of a domain.

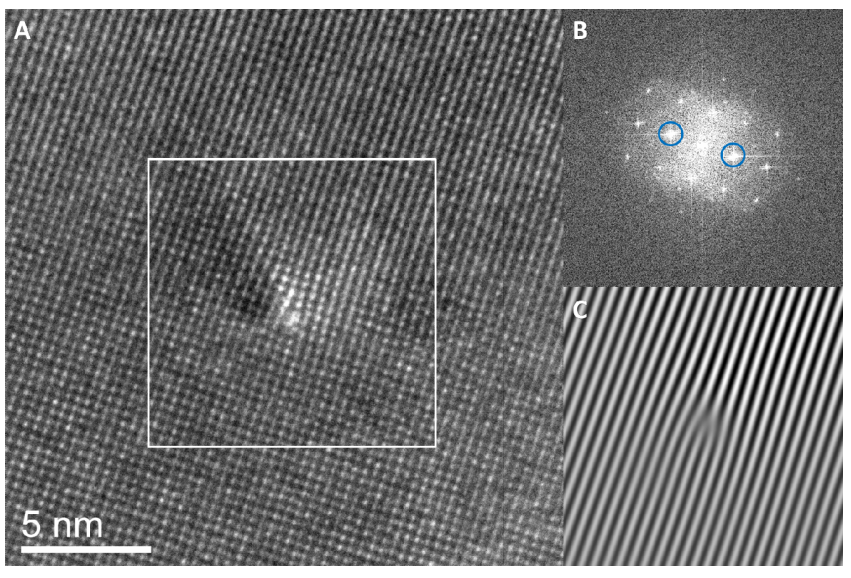

Figure 2: Visualizing an edge dislocation in BFO. A) A high-resolution bright field TEM image of an edge dislocation in BFO. B) The Fourier transform of B). C) The inverse Fourier transform composed from the frequencies circled in B). The edge dislocation is easily identified.

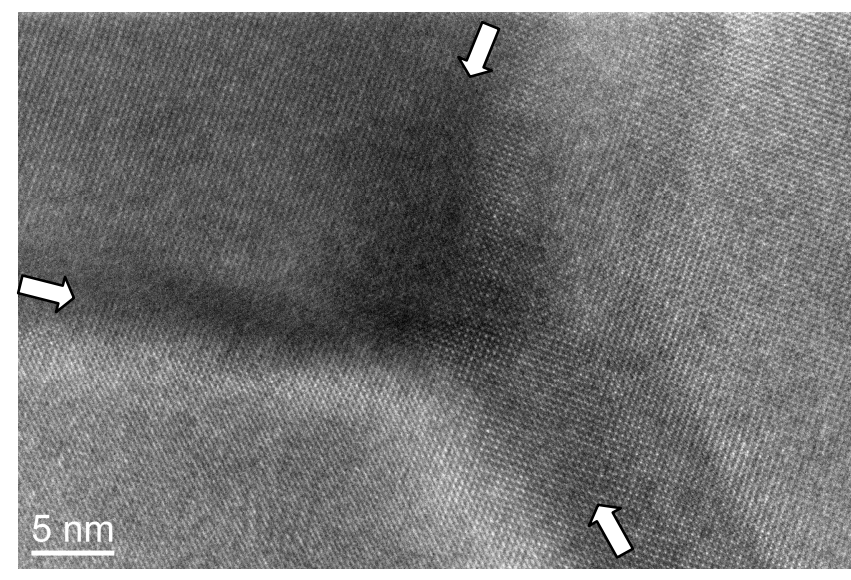

Figure 3: A high-resolution bright field TEM image of the junction between three separate domain walls, indicated by arrows, in BFO. 